\title{
Competing order in the fermionic Hubbard model on the hexagonal graphene lattice
}

\author{
Pavel Buividovich \\ Institut für Theoretische Physik, Universität Regensburg, 93053 Regensburg, Germany \\ E-mail: pavel.buividovich@physik.uni-regensburg.de
}

\section{Dominik Smith}

Institut für Theoretische Physik, Justus-Liebig-Universität Gießen, 35392 Gießen, Germany

E-mail: dominik.smith@theo.physik.uni-giessen.de

\section{Maksim Ulybyshev}

Institut für Theoretische Physik, Universität Regensburg, 93053 Regensburg, Germany

E-mail: maksim.ulybyshev@physik.uni-regensburg.de

\section{Lorenz von Smekal*}

Institut für Theoretische Physik, Justus-Liebig-Universität Gießen, 35392 Gießen, Germany

E-mail: lorenz.smekal@theo.physik.uni-giessen.de

\begin{abstract}
We study the phase diagram of the fermionic Hubbard model on the hexagonal lattice in the space of on-site and nearest neighbor couplings with Hybrid-Monte-Carlo simulations. With pure on-site repulsion this allows to determine the critical coupling strength for spin-density wave formation with the standard approach of introducing a small mass term, explicitly breaking the sublattice symmetry. The analogous mass term for charge-density wave formation above a critical nearest-neighbor repulsion, on the other hand, would introduce a fermion sign problem. The competition between the two and the phase diagram in the space of the two coouplings can however be studied in simulations without explicit sublattice symmetry breaking. Our results compare qualitatively well with the Hartree-Fock phase diagram. We furthermore demonstrate how spin-symmetry breaking by the Euclidean time discretization can be avoided also, when using an improved fermion action based on an exponetial transfer matrix with exact sublattice symmetry.
\end{abstract}

34th annual International Symposium on Lattice Field Theory

24-30 July 2016

University of Southampton, UK

\footnotetext{
${ }^{*}$ Speaker.
} 


\section{Introduction}

Possible insulator-semimetal Mott transitions in the hexagonal Hubbard model with varying on-site repulsion and nearest- and next-to-nearest-neighbor interaction strengths have been a subject of active theoretical studies which led to a rather rich phase diagram with anti-ferromagnetic (AF) spin-density wave (SDW) and charge-density wave (CDW) phases [1, 2, 3], topological insulators [4] and spontaneous Kekulé distortions [5]. While by now it is well established both experimentally [6] and numerically [7, 8] that free suspended graphene is a semimetal, the interest in this rich phase structure is still not purely academic. It is expected that some of these phases can be realized in mechanically strained graphene [9], in other recently discovered 2D materials with hexagonal lattices such as phosphorene [10], silicene and germanene [11] or by designing an "artificial graphene" with optical lattices [12].

While semi-analytic methods such as a large- $N$ renormalization group fixed-point analysis [3] or a variational Hamiltonian approach [13], which is equivalent to solving the Dyson-Schwinger equations on the hexagonal lattice in Hartree-Fock approximation [14], provide a reasonably good qualitative description of the phase diagram in the parameter space of on-site and nearest-neighbor couplings, there are considerable variations in the actual values of critical couplings and the locations of phase-transitions among these methods. In order to produce quantitative results for the phase diagram one should therefore turn to first-principles numerical simulations. The transition separating semimetal and SDW phases in the hexagonal Hubbard model with only on-site interaction has recently been studied in detail in [15] using Determinantal Quantum Monte-Carlo with ground-state projection. Here we present first results for the phase diagram of this model with both on-site and nearest-neighbor interactions from Hybrid Monte-Carlo (HMC) simulations. In contrast to Determinantal Quantum Monte-Carlo, this algorithm avoids the use of explicitly calculated ratios of fermionic determinants in the Metropolis accept-reject step which significantly speeds up the sampling for large lattice sizes [16]. We also demonstrate how to perform simulations with exact sublattice symmetry, by using a gapless single-particle Hamiltonian and a novel discretization of the fermionic action in Euclidean time. In particular, this allows us to study CDW order and its competition with SDW order in the space of on-site and nearest-neighbor couplings.

\section{HMC simulations in the chiral limit of graphene}

Since spontaneous symmetry breaking cannot happen in a system with a finite number of degrees of freedom, detecting it in Monte-Carlo simulations on a finite lattice is a nontrivial numerical problem. The standard strategy also used in our previous HMC studies of the semimetal-insulator transition in suspended graphene $[7,8]$ is to introduce a small explicit mass term $m_{S D W}$ which induces SDW order and thus opens a small gap in the energy spectrum of the single-particle Hamiltonian. The measured SDW order parameter is then extrapolated to infinite volume and $m_{S D W} \rightarrow 0$ in the right order, yielding a nonzero result in the phase where the sublattice symmetry is spontaneously broken by the anti-ferromagnetic SDW ground state.

One of the disadvantages of this method is the need to perform two extrapolations (towards infinite volume and zero mass). Moreover, the corresponding mass term to study CDW order violates the particle-hole symmetry and thus leads to a fermion-sign problem in HMC simulations. 

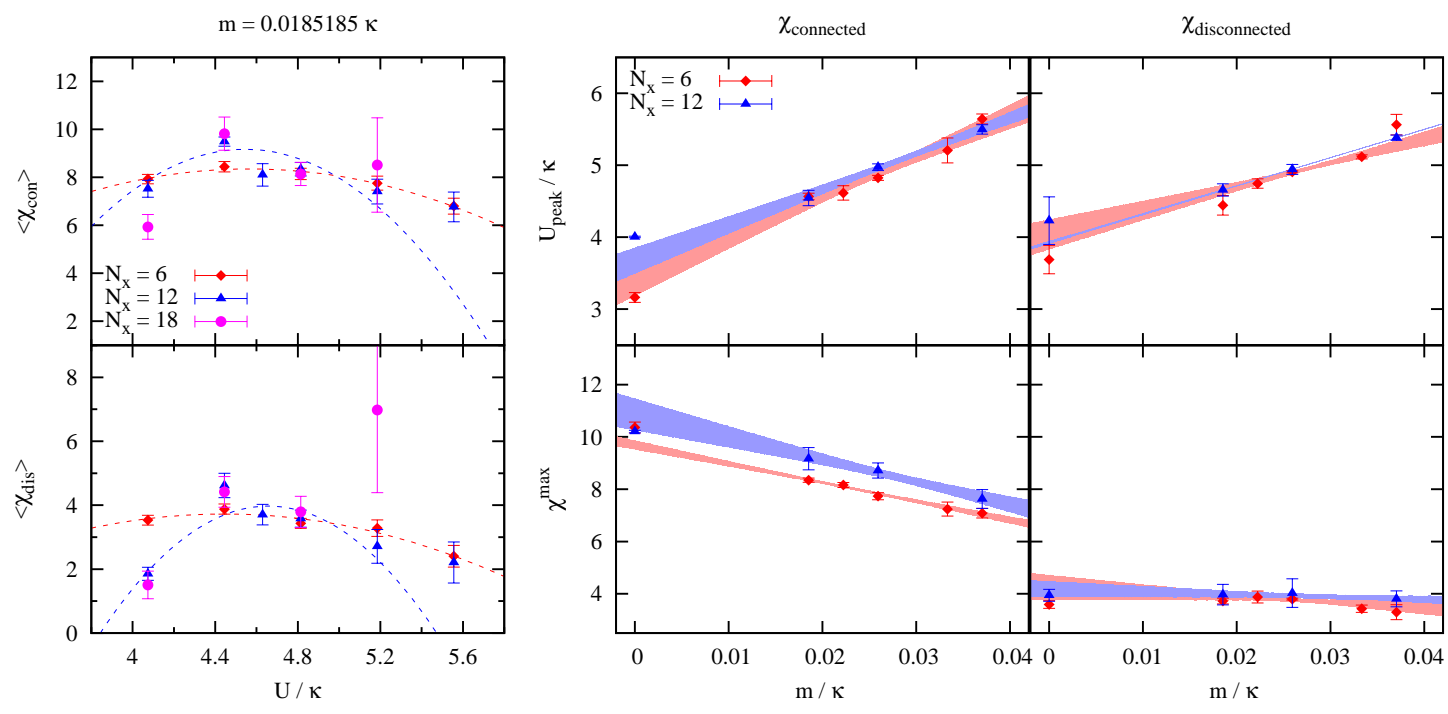

Figure 1: Dependence of connected and disconnected parts of the susceptibility for SDW order-parameter fluctuations on the on-site interaction strength at small but finite mass with interpolations to extract the peaks (left) which are then extrapolated and compared with results from simulations at exactly zero mass (right).

An alternative strategy, for which only the infinite-volume extrapolation is required, is to study the susceptibilities of the relevant order parameters at exactly zero mass. Their peaks can indicate phase transitions even when the latter vanish in a finite volume. To test this, we have verified numerically that the zero-mass extrapolations of the susceptibilities agree with the results of simulating these susceptibilities at exactly zero mass on a finite lattice within errors. The comparison is limited to the case of only on-site interactions of strength $U$ where spin-density wave formation is well established for $U>U_{c}[1,15]$, and simulations with the relevant mass term do not suffer from a sign problem. In the left panel of Fig. 1 we show the dependence of the susceptibility $\chi$ of the SDW order parameter on $U$ for the smallest mass we have used in our simulations. We separately plot the contributions of connected $\left(\chi_{\text {con }}\right)$ and disconnected $\left(\chi_{\text {dis }}\right)$ fermionic diagrams to the susceptibility at different lattice volumes. Both contributions feature clearly visible peaks which indicate the transition point. There is some evidence for a volume scaling indicative of a phase transition, but better statistics on larger lattices will be necessary to confirm that. In the right panel we compare the results of linear extrapolations of positions and heights of the susceptibility peaks with the results obtained directly at zero mass. In all cases the latter are close to the $95 \%$ confidence intervals for linear extrapolations. There is a considerable shift of the susceptibility peaks towards smaller values of $U$ as the explicit SDW mass is reduced, but both, the extrapolated and the measured peak positions at zero mass are overall consistent with previous determinations of the critical coupling $U_{c} \simeq 3.8 \kappa[15]$ in units of the tight-binding hopping parameter $\kappa$.

Finally we notice that in contrast to expectations from lattice QCD, in practice our HMC simulations run quite smoothly even at exactly zero mass. They do not get stuck in some region of configuration space (such as fixed-topology sectors in QCD). The reason most likely is that there is simply no non-trivial topology for the Hubbard fields involved in graphene simulations, and the geometry of manifolds on which the fermion determinant is zero is certainly quite different 
from that in QCD. For simple few-site tight-binding models in the large-temperature limit we have checked explicitly that zeros of the fermion determinant form manifolds of dimension $N-2$ in the $N$-dimensional configuration space of the Hubbard field. It is clear that manifolds of such dimension cannot enclose an $\mathrm{N}$-dimensional region where the molecular dynamics could get stuck. In rare events it can get close to one of these $N$-2-dimensional manifolds which only results in a large and positive molecular-dynamics energy difference, however, and therefore leads to rejection in the Metropolis check.

The possibility of simulations at exactly zero mass can be proven in a more rigorous way by noticing that for lattice sizes which are not multiples of three Dirac points are not within the discrete set of lattice momenta. As a result, the spectrum of the single-particle Hamiltonian on such lattices has a small gap inversely proportional to the lattice size, and the fermion determinant never crosses zero. Thus there are no impenetrable barriers in the HMC dynamics, and the whole configuration space can be efficiently sampled. We call this geometry-dependent energy gap a "geometric mass" term. In contrast to the mass terms which explicitly break sublattice symmetry and induce spindensity or charge-density wave order, the geometric mass term does not break sublattice symmetry and does not favor any order in the ground state. It is thus ideally suited for our purposes.

\section{Phase diagram with on-site and nearest-neighbor interactions}

To identify the phase transitions in the space of combined on-site $U$ and nearest-neighbor repulsion of strength $V$ we follow the approach of [17] and study the volume dependence of expectation values of sums of squares of sublattice averages as order parameters. SDW order, for example, can be identified from averages of squared sublattice magnetizations

$$
\left\langle S^{i}\right\rangle=\frac{1}{L^{2}} \sqrt{\left\langle\left(\sum_{x=(A, \xi)} \hat{S}_{x}^{i}\right)^{2}\right\rangle+\left\langle\left(\sum_{x=(B, \xi)} \hat{S}_{x}^{i}\right)^{2}\right\rangle},
$$

where $L$ is the lattice size, $\hat{S}_{x}^{i}=\frac{1}{2}\left(\hat{a}_{x, \uparrow}^{\dagger}, \hat{a}_{x, \downarrow}^{\dagger}\right) \sigma_{i}\left(\begin{array}{l}\hat{a}_{x, \uparrow} \\ a_{x, \downarrow}\end{array}\right)$ is the spin operator at lattice site $x=(\alpha, \xi)$, where $\alpha=A, B$ is sublattice index and $\xi$ the elementary cell coordinate, and $\sigma_{i}$ are the Pauli matrices. The sum over both sublattices is used to reduce statistical errors. This observable can be easily expressed in terms of fermionic two-point Greens functions. An analogous definition of $\langle Q\rangle$ as (the square root of) the sum of the expectation values of squared electric charges per sublattice can be used to study CDW order at half-filling.

Observables as in Eq. (3.1) can have finite $L \rightarrow \infty$ limits only in phases with the corresponding long-range order. The volume dependence of $\left\langle S^{x}\right\rangle$ in our geometric mass simulations, i.e. without explicit sublattice symmetry breaking, is shown in the leftmost panel of Fig. 2 for selcted values of the on-site coupling $U \equiv V_{00}$ at vanishing $V \equiv V_{01}$. The transition to the AF phase with SDW order is again observed at around $U_{c} \simeq 3.7$, in agreement with the previous section. The middle panel of Fig. 2 shows selected examples with and without CDW order as signaled by a non-zero extrapolation of $\langle Q\rangle$ depending on the strength of the nearest-neighbor repulsion. We have performed analogous simulations with zero mass for a set of points covering the most interesting region of the phase diagram and used infinite-volume extrapolations of $\left\langle S^{i}\right\rangle$ and $\langle Q\rangle$ to identify spontaneous symmetry breaking with SDW or CDW order. 

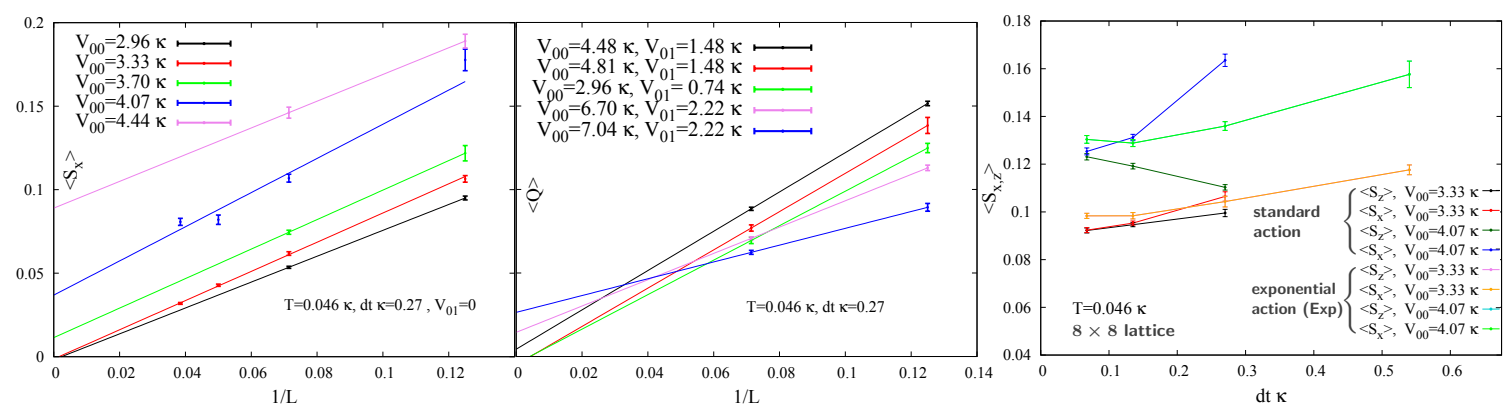

Figure 2: Infinite volume extrapolations of $\left\langle S^{x}\right\rangle$ at vanishing nearest-neighbor coupling $V_{01} \equiv V$ indicating SDW order for $V_{00} \equiv U \geq U_{c} \sim 3.7 \kappa$ (left), and of $\langle Q\rangle$ for various combinations of $U$ and $V$ (middle); comparison of $\left\langle S^{x}\right\rangle$ and $\left\langle S^{z}\right\rangle$ for different Euclidean time discretizations (right), leading to rather strong spin-symmetry breaking in the conventional local fermion action whereas the results for $\left\langle S^{x}\right\rangle$ and $\left\langle S^{z}\right\rangle$ completely coincide with the improved non-local action (4.1) with exact sublattice symmetry.

The resulting numerical estimate of the phase diagram is shown in the left panel of Fig. 3 where red squares and blue triangles mark non-zero infinite-volume extrapolations of the SDW and CDW order parameters, respectively. The black dots mark the points where both extrapolations are consistent with zero as evidence for the semimetal phase. The straight yellow line separates the region with $V>U / 3$ where our HMC algorithm can no-longer be used because the interaction matrix ceases to be positive definite thus invalidating the Hubbard-Stratonovich transfromation [16]. Along this line with $V=U / 3$, due to the coordination number three of the graphene lattice, SDW and CDW ground states have equal interaction energies and one therefore expects this to become a first order transition line in the strong-coupling limit. This is also seen in our HartreeFock (HF) phase diagram from the Dyson-Schwinger equations on the hexagonal lattice [14] which agrees with that of Ref. [13] and is shown in the right panel of Fig. 3. The black first-order transition line in the strong coupling-region of the HF phase diagram ends where the SDW, CDW semimetal phases meet, in what is probably a Lifshitz point (marked in red). The boundary lines of the semimetal (SM) region both mark continuous transitions, most likely in the Gross-Neveu universality class as predicted for the SDW transition $[3,15]$. They continue at larger couplings as spinodal lines limiting possible coexistence regions around the first-order line. Interestingly, for the two points of our data close to this line with the strongest couplings both, the SDW and the CDW order parameter extrapolate to non-zero infinite-volume limits indicating that they might indeed be in the co-existence region. At the moment our HMC data does not allow to determine the order of the transitions between semimetal, SDW and CDW phases. Overall, the phase diagram agrees qualitatively quite well with the expectations from the HF calculation, however.

\section{Spin-symmetry violation by time discretization and improved fermion action}

In absence of spin-dependent interactions, the two physical spin states of the electrons on the hexagonal lattice are treated as two independent fermion fields which can be freely rotated into each other by unitary $U(2)$ transformations analogous to different flavors in QCD. This implies that the squares of all spin components in (3.1) should be equal to one another. In practice, however, one observes that $\left\langle S^{x}\right\rangle$ is significantly larger than $\left\langle S^{z}\right\rangle$, as seen in the right panel of Fig. 2 (without the 

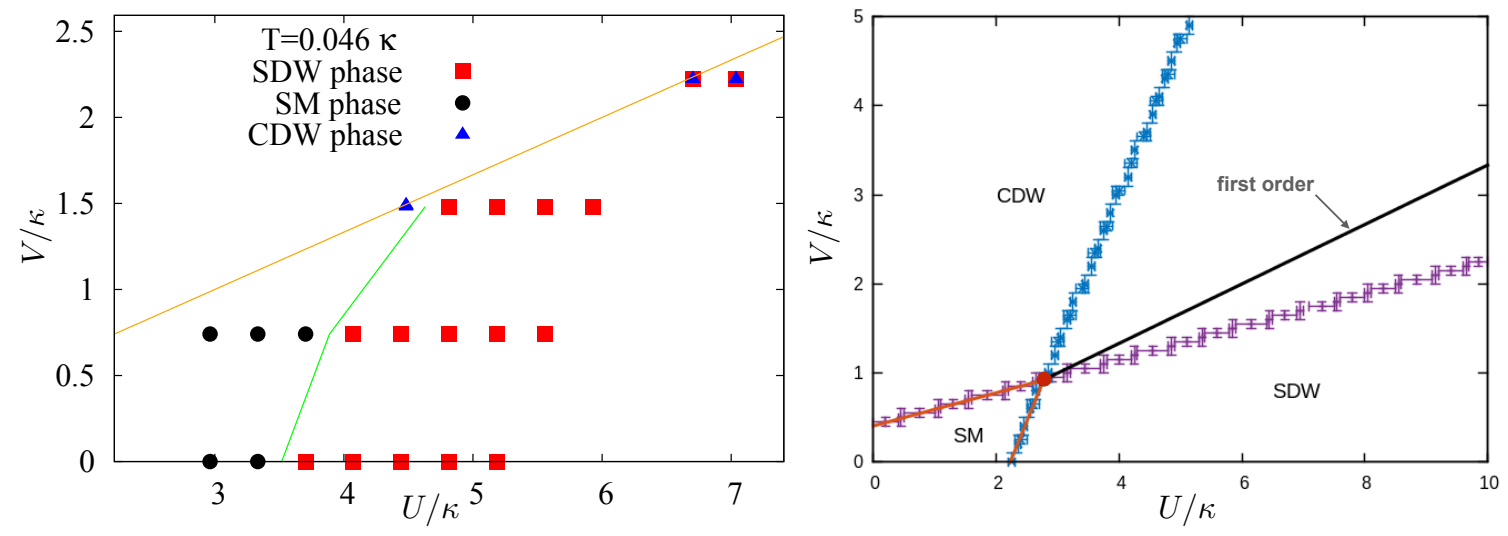

Figure 3: Preliminary phase diagram of the Hubbard model on the hexagonal lattice in the parameter space of on-site $U$ and nearest-neighbor interaction $V$ from the HMC simulations with geometric mass of this work (left) compared to the Hartree-Fock phase diagram (right) from Dyson-Schwinger equations [14].

"Exp" in the label), and that the difference vanishes only very slowly in the contimuum limit. We have simulated lattices with up to $N_{\tau}=640$ time slices to verify that the spin-symmetry does get restored when approaching the continuum limit in the Euclidean time discretization, eventually.

The origin of this asymmetry lies in an identification of physical say spin-up electron states with particles and spin-down with anti-particles which, on one hand, depends on the choice of the basis and, on the other hand, involves a sublattice-dependent phase [7, 8]. This couples spin and sublattice symmetries. In terms of the single-particle Hilbert space one can define a generator $\Sigma_{x y}$ of sublattice symmetry whose non-vanishing matrix elements for $x=y$ are +1 on one sublattice and -1 on the other sublattice. This generator is completely analogous to $\gamma_{5}$ for the Dirac Hamiltonian. In particular, the single-particle Hamiltonian satisfies (in the absence of SDW or CDW mass terms) the identity $\Sigma h \Sigma=-h$. Thus exchanging the two sublattices is equivalent to exchanging positive- and negative-energy states, which are equivalent by virtue of the particlehole symmetry of the bipartite lattice. At the level of the finite-temperature partition function, this identity implies $\Sigma e^{-\beta h} \Sigma=e^{\beta h}$. However, if one discretizes the Euclidean time into intervals of size $\Delta \tau=\beta / N_{\tau}$ and replaces the exponent $e^{-\beta h}$ by $(1-\Delta \tau h)^{N_{\tau}}$, such an identity no-longer holds, since $\Sigma(1-\Delta \tau h)^{N_{\tau}} \Sigma=(1+\Delta \tau h)^{N_{\tau}} \neq(1-\Delta \tau h)^{-N_{\tau}}$. In other words, the particle transfer matrix is no-longer the inverse of the hole transfer matrix after exchanging sublattices, the particle propagating backwards in time is no-longer equivalent to a hole, and thus the combined particle-hole and sublattice symmetries are violated by corrections of order $\Delta \tau$. Since particles and holes were identified with certain spin components, this violation translates into one of the spin symmetry.

To overcome this difficulty we propose to use a spatially non-local fermion action in which the factors $(1-\Delta \tau h)$ are replaced by the exponents $e^{-\Delta \tau h}$,

$$
S\left[\bar{\psi}_{\tau}, \psi_{\tau}, \phi_{\tau}\right]=\sum_{\tau}\left(\bar{\psi}_{\tau} \psi_{\tau}-\bar{\psi}_{\tau} e^{-\Delta \tau h} e^{i \phi_{\tau}} \psi_{\tau+\Delta \tau}\right)
$$

where $\psi_{\tau}$ are the fermion fields at time slice $\tau$ (with spatial indices omitted here), $\phi_{\tau}$ are the Hubbard fields at time slice $\tau$ (interpreted as a diagonal matrix in the single-particle Hilbert space) and $h$ is the free single-particle Hamiltonian of the tight-binding model. One can show that even in the 
presence of fluctuating Hubbard fields this action has an exact sublattice-particle-hole symmetry, and thus restores the symmetry between different spin components.

To illustrate this, in the right panel of Fig. 2 we also show the observables $\left\langle S^{i}\right\rangle$ from Eq. (3.1) for SDW order calculated with the improved fermion action (4.1). The corresponding data points (with the "Exp" in the label) for $\left\langle S^{x}\right\rangle$ and $\left\langle S^{z}\right\rangle$ lie exactly on top of each other which demonstrates the complete restoration of spin symmetry. An additional advantage is that discretization artifacts seem to be much smaller with the improved action (4.1). Being non-local and invariant under chiral sublattice symmetry, the fermion action (4.1) is in fact similar to the overlap action in lattice QCD. The non-locality of the action renders the Conjugate Gradient inversions within the HMC algorithm rather slow. We have therefore implemented alternative inversion algorithms which significantly accelerate HMC simulations with the non-local action (4.1) as will be presented elsewhere.

In summary, we have developed the machinery to study the phase diagram of the hexagonal Hubbard model in the space of on-site and nearest-neighbor coupling. We have identified suitable observables to study the competition between spin-density and charge-density wave order without explicit sublattice-symmetry breaking, and we have implemented an improved fermion action that does not break this symmetry by the Euclidean time discretization either. To reduce the systematic uncertainties we currently repeat the calculations of the phase diagram with the improved action. This work was supported by DFG grants BU 2626/2-1 and SM 70/3-1.

\section{References}

[1] S. Sorella, E. Tosatti, Europhys. Lett. 19 (1992), 699.

[2] G. W. Semenoff, Phys. Rev. Lett. 53 (1984), 2449 - 2452.

[3] I. F. Herbut, Phys. Rev. Lett. 97 (2006), 146401, ArXiv:cond-mat/0606195.

[4] S. Raghu, X. Qi, C. Honerkamp, S. Zhang, Phys. Rev. Lett. 100 (2008), 156401, ArXiv:0710.0030.

[5] C.-Y. Hou, C. Chamon, C. Mudry, Phys. Rev. Lett. 98 (2007), 186809.

[6] A. S. Mayorov, D. C. Elias, I. S. Mukhin, S. V. Morozov, L. A. Ponomarenko, K. S. Novoselov, A. K. Geim, R. V. Gorbachev, Nano Lett. 12 (2012), 4629-4634, ArXiv:1206.3848.

[7] M. V. Ulybyshev, P. V. Buividovich, M. I. Katsnelson, M. I. Polikarpov, Phys. Rev. Lett. 111 (2013), 056801, ArXiv:1304.3660.

[8] D. Smith, L. von Smekal, Phys. Rev. B 89 (2014), 195429, ArXiv:1403.3620.

[9] H. Tang, E. Laksono, J. N. B. Rodrigues, P. Sengupta, F. F. Assaad, S. Adam, Phys. Rev. Lett. 115 (2015), 186602, ArXiv:1505.04188.

[10] H. Liu, A. T. Neal, Z. Zhu, Z. Luo, X. Xu, D. Tománek, P. D. Ye, ACS Nano 8 (2014), 4033 - 4041.

[11] S. Cahangirov, M. Topsakal, E. Aktürk, H. Şahin, S. Ciraci, Phys. Rev. Lett. 102 (2009), 236804.

[12] L. Tarruell, D. Greif, T. Uehlinger, G. Jotzu, T. Esslinger, Nature 483 (2012), 302-305.

[13] Y. Araki, G. W. Semenoff, Phys. Rev. B 86 (2012), 121402, ArXiv:1204.4531.

[14] K. Kleeberg, D. Smith, L. von Smekal, in preparation (2016).

[15] F. F. Assaad, I. F. Herbut, Phys. Rev. X 3 (2013), 031010, ArXiv:1304.6340.

[16] P. V. Buividovich, M. V. Ulybyshev, Int. J. Mod. Phys. A 31 (2016), 1643008, ArXiv:1602.08431.

[17] M. Hohenadler, F. Parisen Toldin, I. F. Herbut, F. F. Assaad, Phys. Rev. B 90 (2014), 085146. 